\title{
EL FIN DE UN MITO: \\ CAUSAS CLÍNICAS DE LA MUERTE DE FERNANDO EL CATÓLICO
}

\author{
The End of a Myth: Clinical Causes \\ of Ferdinand the Catholic's Death
}

Jaime Elipe y Beatriz VILlagrasa BlasCO ${ }^{1}$

Grupo de Investigación «Blancas» / Hospital Clínico Lozano Blesa

\section{Resumen}

El conocimiento sobre Fernando el Católico en la actualidad continúa siendo bastante limitado, especialmente en lo referente a sus últimos días y posterior muerte. Tradicionalmente se achacó su deterioro de salud, según los principales historiadores coetáneos y posteriores, al empleo abusivo de afrodisíacos para poder engendrar al ansiado heredero con la reina Germana de Foix. Pedro Mártir de Anglería fue el único que recogió datos de forma continuada en el tiempo, que han sido pasados por alto por la historiografía. Mediante un estudio interdisciplinar, se ha realizado una lectura sistemática de las cartas del Epistolario del humanista lombardo, extrayendo todas las noticias del progreso de su salud para trazar un cuadro clínico. A partir de este, los afrodisíacos quedan definitivamente excluidos como causa del deterioro de la salud del rey. Tomando los edemas y la disnea como principales síntomas, se ofrece una nueva hipótesis sobre el fallecimiento del Rey: el fallo cardíaco.

Palabras clave: Fernando el Católico, muerte, enfermedad, insuficiencia cardíaca, afrodisíacos

1 Jaime Elipe pertenece al grupo de investigación H01_17R Blancas del Gobierno de Aragón y la Universidad de Zaragoza. Correo electrónico: jelipe@unizar.es. Beatriz Villagrasa es Médico Interno Residente en el HCU Lozano Blesa y colaboradora extraordinaria en el Departamento de Medicina, Psiquiatría y Dermatología. Correo electrónico: beavibla@gmail.com. Este trabajo se inscribe en el Proyecto HAR2014-52434-C5-2 Elites políticas y religiosas, sacralidad territorial y hagiografía en la Iglesia hispánica de la Edad Moderna, del Ministerio de Economía y Competitividad del Gobierno de España. Fecha de recepción del artículo: 14 de noviembre de 2017. Fecha de aceptación: 30 de enero de 2018. Versión final: 30 de junio de 2018. 


\section{Abstract}

Nowadays, the knowledge about Ferdinand the Catholic is quite limited, especially on his last days and his death. According to the main historians, the obit of the king was caused by the massive use of aphrodisiacs due to the desire of fathering an heir with Germaine of Foix. The works of Pedro Mártir de Anglería offers much more data about the evolution of Ferdinand's disease that was never kept in mind. Through an interdisciplinary study, a systematic reading of the Epistolario letters of the lombard humanist has been carried out in order to describe a medical condition. Based on it, the thesis of abuse of aphrodisiacs has to be discard completly. As the oedemas and dyspnoea are the principal sytoms, a new hypothesis about the death of the Catholic King is offered: the heart failure.

Key words: Ferdinand the Catholic, death, illness, heart failure, aphrodisiacs

\section{INTRODUCCIÓN}

Hace quinientos años, el humanista Pedro Mártir de Anglería tomaba la pluma como cada día para escribir a sus allegados. Sus cartas versaban sobre los más distintos temas, por lo general de los asuntos políticos europeos y de las idas y venidas en la corte, donde residía. En esta ocasión, su interlocutor era Luis Marliano, obispo de Tuy, a quien transmitía el fallecimiento de Fernando el Católico. Tal y como lo describía, este «amplificador de la religión cristiana y domeñador de sus enemigos», había fallecido «en una rústica casa y en la pobreza» (Anglería, 1956: 217). Efectivamente, el rey de Aragón acababa de morir en una casa humilde del pequeño pueblo extremeño de Madrigalejo, cuando marchaba a presidir el capítulo de la Orden de Calatrava. El suceso no fue ninguna sorpresa, ya que el monarca había visto 63 inviernos y desde hacía varios años, su salud se hallaba muy quebrantada.

La muerte de los reyes es algo que desde la Antigüedad ha despertado curiosidad entre los historiadores, especialmente si esta se producía de manera desagradable y violenta. Un ejemplo paradigmático fue Suetonio y su Vida de los doce césares, quien se recreaba en la desaparición de los distintos principes romanos del Alto Imperio. Es evidente que el fallecimiento real era un momento clave para los estados y de ahí el interés de la Historia por los mismos. Este evento, en cualquier época, traía consigo multiplicidad de cuestiones, siendo la sucesión la primera de todas. La muerte del soberano, según cómo se hubiera producido — tranquila, repentina, violenta-, se tornaba elemento capital a la hora de entender los sucesos his- 
tóricos posteriores. Sin embargo, el camino hasta el deceso del monarca casi nunca ha despertado el interés de los estudiosos. Un regicidio siempre es explicado desde sus inicios, cómo se urdió el complot, quiénes lo llevaron a cabo, qué les movió a ejecutarlo, etc. No así ha venido sucediendo cuando un rey moría por causas naturales; bastaba con citar la causa, por ejemplo unas fiebres o edad muy avanzada. ${ }^{2}$

En las antesalas de la llegada de la Casa Habsburgo a las Españas se encuentra una complicada enfermedad sobre la que poco se conoce hasta hoy: la que condujo a la muerte a Fernando el Católico (1452-1516). A todas luces, sus tres últimos años de vida estuvieron marcados por una salud en franco deterioro que, al parecer, acabó por afectarle anímicamente $\mathrm{y}$ trastocar ciertos aspectos de su personalidad. ${ }^{3}$

\section{ESTAdo DE LA CUESTIÓN Y FUENTES}

En ocasiones, la enfermedad que sufrían los reyes y que finalmente acababa con sus días, podía prolongarse bastante en el tiempo. No estamos hablando de semanas, o meses, sino de años enteros. Es cierto que apenas ha habido casos en la historia de España en los que se haya padecido durante años serias enfermedades. Posiblemente el caso paradigmático sea el conjunto de la vida de Carlos II. Sus problemas congénitos son más que conocidos; estar bechizado fue fruto de la compleja política endogámica de los Habsburgo

2 La muerte de los reyes ha interesado siempre, pero por desgracia, también la atención se ha prestado de manera habitual a aquellos que la han sufrido violentamente. Sin ánimo de ser exhaustivos, obras de referencia para el período medieval son Martín Rodríguez (1991) y Pérez Monzón (2007). Por su parte, para la Edad Moderna, los estudios se han centrado en las representaciones de la muerte de los monarcas, principalmente en sus exequias, en la línea de la Historia Cultural.

3 Aunque escapa al objetivo principal del presente artículo, las manifestaciones psicológicas de la enfermedad a las que Pedro Mártir de Anglería hizo alusión, podrían considerarse como reactivas a la situación de enfermedad en una persona considerada como «sana» hasta entonces y que comenzaba a ser consciente de sus limitaciones. Los síntomas de irritabilidad, apatía y falta de concentración a los que el lombardo hizo alusión, fácilmente podrían considerarse como un trastorno adaptativo a esta nueva situación. No da la impresión, a nuestro parecer, que pudiera haber existido patología psiquiátrica genuina. En cuanto a la supuesta catarsis de celos que sufrió con el vicecanciller Agustín, tenía un componente político que descarta cualquier tipo de enajenación. Por último, acerca del «horror a vivir bajo techado", de aparición reciente a sus 61 años, no parece que se deba a una fobia sino más bien se encontraba en relación con la patología orgánica que presumiblemente presentaba. 
(Álvarez, Ceballos y Quinteiro, 2009). Como toda su vida fue un cuadro clínico complejo y afectó directamente al gobierno de la Monarquía Hispánica, en este caso enfermedad y persona fueron indisolublemente asociados.

El primero de los trabajos dedicado al diganóstico de enfermedades de los monarcas hispanos es el de la muerte del príncipe don Carlos, primogénito de Felipe II. Sanchís Banús lo publicó en 1927 y en este se concluía tras un método riguroso que su objeto de estudio había sufrido esquizofrenia paranoide (Sanchís Banús, 1927). Evidentemente, se trataba de un caso muy interesante y controvertido por ser una de las inspiraciones principales de la Leyenda Negra. Más extenso y famoso fue el libro de Gregorio Marañón en 1941 sobre Enrique IV, en el que estudiaba la casuística ambiental a la que estaba sometido y a muy distintos aspectos de su época, si bien también hacía juicios de valor sobre moral, psicología o aspectos sociales. Aparte de ofrecer un abanico de posibilidades sobre su fulminante muerte -en tan apenas dos días-, el famoso endocrino también dejó algunas reflexiones sobre lo que él denominaba la «clínica arqueológica» (Marañón, 1997: 49). Algún otro estudio sobre el deceso de los monarcas es uno bastante breve sobre el óbito de Fernando I de Aragón (Vendrell de Millás, 1958). Avanzando en el tiempo, aunque siguiendo una línea de estudio similar, se encuentran análisis clínicos francamente profundos pero que suelen presentar un insuficiente trato de las fuentes históricas (Gómez González , 2004; Guerrero Peral, 2009). Ya en época reciente se encuentra el estudio de la momia de Sancho de Castilla, de carácter completamente distinto a los anteriores por su multidisciplinariedad y la posibilidad de usar métodos empíricos en laboratorio (Franco, Esteban et al., 2012). En una vertiente menos experimental se encuentra un artículo sobre los fallecimientos de los reyes de Castilla, Margarita Cabrera tan apenas se detiene en la muerte del Católico por dedicarles mayor espacio a otros miembros de la realeza (Cabrera Sánchez, 2011).

Atendiendo a la figura de Fernando II, este ha recibido cierta atención, ya sea de forma específica o dentro de un elenco con otros monarcas. Sin embargo, su solvencia científica es más que discutible. Existe una Patobiografía centrada en exclusiva en este soberano (Otero Tejero, 2013), en la que se describe de forma superficial el cosmos del Rey Católico, así como la medicina en su época; no profundiza en detalles y las fuentes manejadas son escasas, por lo que las conclusiones son muy limitadas. Por otra parte, la principal obra sobre los reyes y sus dolencias de la Edad Moderna es de Pedro Gargantilla; esta presenta un carácter divulgativo y carece de un aparato crítico contrastado (Gargantilla, 2005). 
Teniendo en cuenta estos precedentes, lo que se pretende con el presente trabajo es, mediante un trabajo interdisciplinar determinar qué es lo que produjo en el Rey Católico el quebranto de su salud durante sus últimos años, y finalmente, su muerte. Para ello, como algunos autores afirman, existen dos tipos de fuentes: los restos arqueológicos - momias, restos óseos-y los materiales creados por el hombre - textos, pintura, escultura- (Mitchell, 2011: 81). En el caso presente, se ha optado por la segunda opción. En este sentido se ha efectuado una búsqueda sistemática y una lectura rigurosa de las distintas fuentes disponibles, con el fin de disponer del mayor número de datos fiables. Con estos, se ha elaborado un cuadro clínico, descartando antiguas teorías - completamente acientíficas- y proponiendo una hipótesis sólida a partir de las fuentes manejadas. Fernando II ha sido un personaje que pese a su fama internacional y haber sido objeto de numerosos trabajos literarios de todo tipo — de carácter político, principalmente-, permanece desconocido en muchos aspectos de su vida. Esto mismo no es ninguna novedad, puesto de relieve en distintas ocasiones por diversos historiadores (Kamen, 2014: 16).

Ahora bien, se es consciente de que existen bastantes problemas a la hora de acercarse a la enfermedad de la que nos separan cinco siglos. El propio Marañón reconocía ya en fechas tempranas que si los médicos podían errar el diagnóstico con sus pacientes, era más fácil que esto ocurriese con personajes históricos (Marañón, 1997: 49-52). Al emplear fuentes literarias, las segundas en la clasificación citada de Mitchell, nos acercamos a una «representación de la realidad, no la realidad en sí misma» a partir de la medicina altomoderna. Además, uno de los principales puntos peligrosos de la investigación radica en el léxico empleado en torno a 1500 y los riesgos que entraña el realizar un ejercicio de «traducción cultural» (Arrizabalaga, 2006: 242-243). El investigador Jon Arrizabalaga ha reflexionado sobre estos asuntos en distintos trabajos y ya hace más de veinte años señalaba la existencia de una casuística muy variada a la hora de determinar la muerte, sobre todo por el bajo grado de medicalización de las sociedades precedentes. De hecho, se ha mostrado crítico con los diagnósticos retrospectivos por los fines perseguidos en investigaciones de bajo valor científico. ${ }^{4} \operatorname{Sin}$

4 Arrizabalaga (1994: 40): «Pese a que en las últimas décadas se aprecia una cautela creciente hacia el diagnóstico retrospectivo, su práctica continúa teniendo cierto impacto en la historiografía de la enfermedad, y muchos investigadores continúan, de modo consciente o inconsciente, integrándola como parte habitual de su discurso. Además, en algunos campos de estudio el diagnóstico retrospectivo se ha prodigado hasta el punto de consti-

STVDIVM. Revista de Humanidades, 24 (2018) ISSN: 1137-8417, pp. 41-60 
embargo, estas aseveraciones se dirigían sobre todo a enfermedades de carácter infeccioso - grandes epidemias-, no a las de personas particulares y dolencias comunes (Arrizabalaga, 2002: 67). Mitchell sostiene una visión bastante más positiva sobre el interés que tienen estas investigaciones, que para no estar limitadas - y desechadas - por marcos conceptuales, propone equipos multidisciplinares para poderlo sortear en la medida de lo posible (Mitchell, 2011: 84).

Las crónicas que narraron los últimos años del Rey Católico adolecen una carencia prácticamente total de datos médicos continuos que puedan ser empleados en una interpretación seria de la enfermedad. Si reseñaron las causas de la muerte lo hacen únicamente en el apartado del fallecimiento - esto es, no antes a enero de 1516- y sin conocer la evolución previa que había podido tener el monarca. Por ello mismo, es necesario emplear como fuente principal el Epistolario de Pedro Mártir, ya que ofrece un buen número, en comparación con otros escritos, de noticias sobre la salud de Fernando II. En esta obra recopiló distintas cartas escogidas a lo largo de varios decenios. Ofrecía desde erudiciones latinas, anécdotas de palacio, batallas, monstruos sobrenaturales o cualquier chisme que pudiera interesar a sus interlocutores, por lo general, antiguos discípulos suyos y humanistas prestigiosos. Las obras de los historiadores Lorenzo Galíndez de Carvajal (1472-1528), Jerónimo Zurita (1512-1580) y fray Prudencio de Sandoval (1552-1620) pueden ser complementarias, pero no se les puede conceder - para este caso en concreto- el mismo grado de veracidad que al humanista lombardo (Galíndez de Carvajal, 1851; Zurita, 2003; Sandoval, 1846). Por lo general, tendieron a copiar las epístolas de Pedro Mártir o directamente a recoger invenciones. También hay que añadir a otro gran escritor, el veneciano Marino Sanudo el Joven (1466-1536). Este, autor de los Diarios, es de interés por ser sus corresponsales cercanos a la corte aunque no por ello su información ha de ser más fiable. ${ }^{5}$ Sin embar-

tuir un fin en sí mismo. Tal es el caso de las patobiografías históricas, un área de trabajo que, en razón de la espectacularidad de algunas de sus conclusiones, posee con frecuencia una «visibilidad» social elevada, pese al escaso nivel de muchas de estas investigaciones».

5 Los diarios de Marino Sanudo fueron la obra magna de este polígrafo. Recoge toda la información recibida en la Serenísima República durante el período comprendido entre 1496 y 1533. Debido a los múltiples intereses de Venecia, se convierte en una crónica europea de primer orden. Fueron editados a finales del XIX y por desgracia permanece apenas conocida. Aunque por lo general ofrece juicios de valor y noticias de notable precisión, respecto al deceso del Católico es justo lo contrario. Véase Sanudo (1887: 510): «Et poi. per letere di 9. Scrive aver mandato il suo secretario dal reverendissimo Santa 
go, tan apenas ofrece datos sino del momento de su muerte. Es importante señalar que al encontrarnos en los primeros momentos de la Modernidad, no se puede contar con «relaciones de sucesos», precursoras de gacetas y periódicos. A partir del siglo XVII, sí existe documentación suficiente para poder tratar las enfermedades de los principales personajes del momento; desgraciadamente no era así a principios del siglo XVI (Peñasco, 2013: 115117).

Pedro Mártir de Anglería fue un clérigo de origen lombardo que pasó a España bajo la protección del II conde de Tendilla, Íñigo López de Mendoza. Desde 1487 estuvo siempre en el ambiente áulico siguiendo a los reyes, exceptuando el período de su embajada a Egipto. El epistolario que publicó se convierte así en una obra preciosa, ya que permite al lector conocer los pormenores cotidianos con una periodicidad más que aceptable. Sus cartas registraron una fracción - desconocemos qué porcentaje- de los males físicos y psicológicos que padeció Fernando el Católico. Así, en el transcurso de tres años, entre enero de 1513 y enero de 1516, se conservan una veintena de noticias referidas a su salud, de distintas cualidades. En ocasiones no se trata más que de un par de líneas y en otras puede ser una larga descripción de un problema concreto, tanto de síntomas como comportamientos. Para obtener la información necesaria para el estudio, ha sido requisito consultar una por una las distintas epístolas. Aunque era conocido el empeoramiento de Fernando II a partir de 1513, había que conocer si previamente escribía a sus corresponsales sobre el estado de salud de los reyes. El ejemplo del intento de asesinato de Barcelona a finales de 1492 y el fallecimiento de Isabel son dos buenas referencias, en las que se puede constatar que hablar de la salud regia no era algo recurrente para él a no ser que fuese de extrema importancia. Como norma general, la documentación conservada en los archivos es de carácter oficial, por lo que tan apenas se pueden encontrar noticias privadas. Precisamente por ser miembro activo del entorno áulico del Católico quedan descartadas las posibilidades que le restarían veracidad que señala Mitchell en su obra sobre el diagnóstico retrospectivo — transmisión, exageración o mal copista-(Mitchell, 2011: 81-82). Ahora bien, la distinta cultura sigue siendo un aspecto pendiente que tratar con prudencia. De lo que no cabe duda alguna es que, Pedro Mártir, a pesar de no ser un médico es una fuente perfec-

Maria in Portico a saper di novo. Li disseaver di Spagna da lo episcopo Butigario suo orator, di 23 Zener, come il re di Spagna era morto. Et a dì 17 si maloe, a d'20 a Guadalù li soprazonze fluxo di sangue, et a di 22 venendo 23 , la note, morite».

STVDIVM. Revista de Humanidades, 24 (2018) ISSN: 1137-8417, pp. 41-60 
tamente fiable, como lo pudo ser Guillermo de Tiro en su momento - tutor de Balduino IV que le diagnosticó la temprana lepra que padeció. ${ }^{6}$ Aunque no hemos podido trazar vínculos con los principales médicos cortesanos, es de esperar que los frecuentó debido a sus muy variadas inquietudes culturales.?

\section{Desarrollo Clínico}

Como hemos indicado, el deterioro de Fernando II tuvo comienzo prácticamente tres años antes de su muerte, en marzo de 1513. A principios de mes, se suponía que el monarca habría ingerido una mezcla afrodisíaca suministrada por su esposa la reina Germana de Foix con la intención de mejorar su potencia sexual y así poder quedar encinta.

El seguimiento, así como la primera noticia, la ofrece Pedro Mártir de Anglería en su epistolario. Informaba de que el día 10 de marzo de 1513 el rey no se encontraba bien y un par de días más tarde vomitó todo lo que había ingerido (Anglería, 1956: 107). Tras este episodio, lo que parecía que había sido algo puntual, se tornó más serio, ya que el 20 de abril su estado revestía cierta gravedad, acusando una «fiebre desconocida» por la cual tuvo que tomar «una medicina y tuvo delirios» (Anglería, 1956: 110). Estas fiebres lo tuvieron completamente apartado de la vida pública y de sus desempeños cotidianos hasta mayo, algo que había sido inusual hasta el momento. ${ }^{8}$ Sí que hay constancia que desde primeros de

6 Mitchell (2011: 84): «It has been argued that retrospective diagnosis should only be attempted when the quality of the sources allow it, when the research team has sufficient understanding of the language, literature and cultural context to interpret the source appropriately, and when the research team knows enough about disease to determine which condition most closely matches the symptoms and signs described in those texts. This leaves us to ponder what represents a good quality source, sufficiently clear to allow retrospective diagnosis. This means a source of information that is as close as possible to giving all the observed signs and symptoms with as little influence as possible from the cultural context. To a degree this will be dependent upon the personality and experiences of the person writing the text, as well as the social environment».

7 Los principales trabajos sobre los médicos de los Reyes Católicos son ya clásicos y ofrecen más un cursus honorum que las relaciones humanas que mantuvieron. Para ello, véase Torre (1944) y Alonso Cortés (1951), siendo este último el más completo —sobre los doctores De la Reina y De la Parra.

8 Un ejemplo de esta afirmación se encuentra en Archivo de la Corona de Aragón, Real Cancillería, reg. 3676, f. 259r: «El Rey. Diputados vuestras letras de vint de Março y doze 
abril su situación había ido mejorando paulatinamente, tal y como había sido informado su hijo don Alonso de Aragón, si bien no directamente por el rey. ${ }^{9}$

Pese a que la crisis remitió poco después, ya no volvió a gozar de la vitalidad que lo caracterizaba; de hecho, en otoño del mismo año, aunque se desconocen los síntomas físicos, el monarca se encontraba entristecido por su delicada salud. Hasta tal punto había sufrido cambios en su carácter, que Pedro Mártir mostraba su preocupación: en octubre no tenía ni «el mismo semblante, ni la misma atención para escuchar ni la misma amabilidad» (Anglería, 1956: 134). Un mes más tarde, en noviembre, el humanista hizo referencia al que consideramos síntoma guía en el deterioro físico del Rey Católico: la disnea o dificultad respiratoria. En este preciso instante, el lombardo atribuyó el deterioro de la salud del rey a algo consumido en marzo, es decir, cuando tuvo los vómitos y posteriormente las fiebres (Anglería, 1956: 138-139).

El nuevo año no trajo al rey reposo en su empeorado estado, sino que añadió a ello molestias gástricas, "callos en el estómago», y ardor. Estos podrían considerarse como dispepsia y que al parecer Fernando II llevaba largo tiempo padeciéndolos. Se vino a sumar a su malestar la muerte del secretario Miguel Pérez de Almazán, compañero y fiel servidor durante casi toda su vida. Pero no únicamente estos males consumían la vida del rey a ojos del cortesano Pedro Mártir. Según él, y era un pensamiento común, el rey abusaba de las que eran en esos momentos sus dos grandes pasiones: la caza y la reina. A tal punto llegaba que «si no se desprende de dos apetitos, muy pronto entregará su alma a Dios y su cuerpo a la tierra» (Anglería, 1956: 162-163).

de Abril hauemos recebido a las quales por la indisposicion de nuestra persona de que ya a dios gracias stamos bien conualecido no se vos pudo antes de agora responder y visto el daño que dezis resulta a esse general». Fernando II a los diputados del reino de Aragón, el 2 de mayo de 1513.

9 Véase Real Academia de la Historia, Colección Salazar y Castro, A-14, f. 137r: «Secretario my muy special amygo. Yo os agradezco la buena nueua que me disteys de la salud y contynuacion de la mejora de la magestat del rey my señor, plega a nuestro señor dyos y a su bien auenturada madre que muchos y muchos años lo guarden y den syempre plazer y alegrya, pues con su vyda bybe todo el mundo y mas nosotros sus subdytos y vassallos. En gradissima manera me haueys obligado con la solycitud y contynuos auysos de la dysposicion de la yndisposicion de su real magestat y assy os pydo sy haueys de hazer por my sea en segyrlo como hastaque lo haueys fecho». Alonso de Aragón, arzobispo de Zaragoza a Miguel Pérez de Almazán, el 13 de abril de 1513. 
Dejando estas impresiones personales y habituales de la época, ${ }^{10}$ en noviembre de 1514 la dificultad respiratoria volvió a ser motivo de preocupación. Además, apareció otro de los síntomas relevantes en el deterioro físico del monarca, los edemas, así descritos: «empeora su asma y el humor que lo va hinchando» (Anglería, 1956: 166).

Un mes más tarde el cuadro clínico del rey estaba lejos de mejorar, ya que «progresa la hidropesía, y ni con el movimiento ni con el reposo disminuye el mal, que poco a poco se va extendiendo» (Anglería, 1956: 169). Las epístolas publicadas de Pedro Mártir no vuelven a mencionar el tema de la salud del Rey Católico en unos cuantos meses, por lo que se puede intuir que no hubo cambios notables en su estado. Únicamente criticaba con amargura su afición a la caza que, a pesar de sus dolencias, practicaba con fruición. Finalmente, el 18 de julio de 1515 tuvo lugar una crisis aguda que estuvo a punto de costarle la vida al monarca aragonés. Por la noche padeció un episodio de disnea aguda, el cual describe Pedro Mártir con fuerte patetismo:

«casi quedó ahogado mientras dormía. Un síncope y el catarro le obstruyeron las fibras del corazón (...) sintió al soberano atragantarse y dar unos horribles ronquidos (...). Acudió al estrépito que formaba el Rey, casi a punto de expirar. Entró en el aposento y lo encontró medio muerto, con la cabeza colgando fuera de la cama. (...) Friccionan sus miembros y sacuden todo su cuerpo. Traspuesto con el habla perdida, torcía los ojos. Le rocían el rostro con agua fría. Por fin volvió en sí». ${ }^{11}$

Esta severa crisis, fue evitada por un montero de Espinosa. Los problemas se sucedieron con bastante rapidez a partir de este momento. Tan sólo un mes más tarde, el cortesano lombardo volvió a hacer alusión a la dimensión psicológica de la enfermedad y comentaba las sospechas hacia su esposa y el vicecanciller de Aragón. Tras haberlo encarcelado por esa supuesta catarsis de celos —opinión de poca consistencia—, ${ }^{12}$ durante el

10 Por ejemplo, la muerte del príncipe Juan, de quien popularmente se pensaba que había fallecido por «exceso de amor».

11 Véase Anglería (1956: 181-182).

12 Aunque esta opción ha sido tomada como válida, los auténticos motivos hay que buscarlos en las Cortes de 1515, con una fuerte confrontación de la nobleza contra el resto de estamentos y el rey. Respecto al vicecanciller Antonio Agustín, afirmaba Dormer (1697: 58): «disponia las cosas por su voluntad, atendiendo mas a la pretension de los Barones, que a conservar y adelantar la Real preheminencia, en que fue severisimo el Rey, mostrandolo con tan grandes resoluciones como parecen de todo su Reynado». 
otoño las preocupaciones del rey discurrían por otros derroteros, ya que Fernando II era incapaz de tener una decisión estable. Una única cosa tenía clara y era un auténtico "horror a vivir bajo techado", por lo que prefería siempre andar por bosques y al aire libre sin importar las inclemencias del tiempo. ${ }^{13}$ En este punto, la información ofrecida por el Epistolario se limita a reseñar su empeoramiento y la sensación de muerte inminente del rey. Finalmente, el deceso tuvo lugar el 23 de enero de 1516.

\section{HiPÓTESIS DIAGNÓSTICAS}

Con base principalmente en el testimonio privilegiado de Pedro Mártir de Anglería, hemos esbozado sucintamente la historia clínica de los últimos años de la vida de Fernando II. La disnea — dificultad respiratoria, lo que se denomina como «asma»- y los edemas — «hidropesía»- apuntan como los síntomas más relevantes. Por lo tanto, a partir del análisis de los mismos, se intentará realizar una reflexión acerca de las causas posibles del deterioro físico del Rey Católico que acabaron finalmente con su vida.

Lo primero de todo es preciso señalar que se trató, según las fuentes consultadas, de un deterioro de aproximadamente dos años y tres meses de evolución —desde que apareció la dificultad respiratoria hasta la defunción-y progresivo ya que no hubo objetiva mejoría desde el comienzo.

\subsection{Afrodisíacos}

La muerte del Rey Católico fue achacada en la época a unos afrodisíacos, exactamente a los testículos de toro. ${ }^{14}$ Sin embargo, no está demostrado que los testículos de toro ocasionen tal deterioro en la salud, aparte de clínica digestiva (náuseas, vómitos...) en caso de ser ingeridos en mal estado. Bien es cierto, y esta es la versión más extendida, que el monarca podría haber ingerido junto a los testículos de toro un compuesto,

13 Sobre la indecisión y agitación, véase Anglería (1956: 184, 198, 201, 204). Un ejemplo ilustrativo, a 28 de octubre de 1515, decía así, p. 198: «Como se va agravando su enfermedad, no sabe el Rey ni dónde dirigirse ni qué es lo que quiere». Prácticamente un mes antes de morir el Rey Católico, el 12 de diciembre, Pedro Mártir de Anglería escribía que «con el pretexto de la caza del ciervo, desea estar siempre a cielo raso, teniendo horror a vivir bajo techado".

14 Véase Anglería (1956: 138-139). Esta noticia, como se ha apuntado al inicio del trabajo, fue repetida posteriormente por otros historiadores. 
es decir, un brebaje. ${ }^{15} \mathrm{El}$ de uso habitual en su época era la 'cantaridina', $\mathrm{y}$ es al que se atribuye normalmente el haber acabado con la vida del Católico (Otero, 2013: 20-21; Gargantilla, 2005: 77). Tanto estas aseveraciones recientes como los de historiadores del siglo XVI carecen de cualquier base empírica.

La cantaridina es una sustancia producida de forma natural por escarabajos pertenecientes al orden Coleoptera, familia meloidae. De las más de 2.000 especies de esta familia, la Cantharis vesicatoria (Lytta Vesicatoria) es conocida como cantárida o en la literatura anglosajona como Spanish fly - mosca española- La cantaridina es una sustancia defensiva para estos insectos, de las llamadas vesicantes, sustancias que en contacto con la piel producen irritación y ampollas. Se obtiene desecando, triturando y pulverizando estos escarabajos. Desde la antigüedad se ha utilizado la cantaridina como afrodisíaco debido a su capacidad para causar congestión vascular e inflamación del tracto genitourinario lo que conduce, cuando se administra en dosis elevadas, a priapismo —erección mantenida en el varón-y congestión pélvica en la mujer.

La cantaridina presenta unos niveles elevados de toxicidad y se estima que la dosis letal para adultos oscila entre 10 y $80 \mathrm{mg}$, aunque en la mayoría de publicaciones se recoge que es inferior a $60 \mathrm{mg}$. La mayor parte de sus efectos tóxicos están relacionados con su propiedad vesicante, al producir ampollas en la piel y mucosas con las que entra en contacto. La intoxicación por cantaridina no es muy frecuente, de hecho hay menos de 30 casos descritos desde 1900 hasta 1970 (Presto y Muecke, 1970: 591592). La mayoría de las intoxicaciones se produjeron como consecuencia de la ingesta por sus propiedades afrodisíacas, administrada en la mayoría de las ocasiones por la pareja, aunque también han sido descritas intoxi-

15 El primero en mencionarlo, y de quien lo tomaron otros historiadores fue Galíndez de Carvajal (1851: 333-334): «En este año por el mes de marzo adolesció el Rey Católico en Medina del Campo viniendo de Carrioncillo, tierra de Medina del Campo que se habia ido a holgar con la Reina Germana su muger, de un potage frio que le hizo dar la dicha Reina, porque le hicieron entender que se haria preñada luego; a lo cual se halló Doña María de Velasco, muger de Juan Velázquez de Cuéllar, de la cual enfermedad al cabo ovo de morir el dicho Rey Católico.» Jerónimo Zurita afirma que esto es lo que ya decían Pedro Mártir de Anglería o Carvajal; Zurita (2005: lib. X, cap. LV): « [...] según Pedro Mártir, y el doctor Carvajal escriben, tuvo ocasión de un feo potaje, que la reina le hizo dar para más habilitarle, que pudiese haber hijos [...] »; SANDOVAL (1846, p. 178): « [...] lo que muchos creyeron, que aquel potaje de la reina Germana le dió para hacerle potente, le postró la virtud natural.» 
caciones por exposición cutánea, inoculación accidental o ingesta de escarabajos —esta en particular, en niños- (Moed et al., 2001).

Esta sustancia afecta a múltiples órganos y sistemas tanto por sus propiedades irritativas como por su mecanismo de acción a nivel celular (potente inhibidor de las proteínas-fosfatasas tipo 1 y tipo 2A). Al ser ingerida, a nivel de tracto gastrointestinal lo más frecuente es ardor y la formación de ampollas en la boca, lengua y orofaringe, la disfagia asociada — dificultad para tragar-, calambres abdominales, vómitos y hematemesis —vómito con sangre- . En mayores cantidades, se ha documentado hematoquecia y tenesmo rectal; en algunos casos puede llegar a afectar al hígado. A nivel genitourinario - ya que la cantaridina se excreta por el riñón-, suele ocasionar dolor lumbar, poliuria, disuria y hematuria que pueden persistir durante más de 15 días. La proteinuria también se desarrolla con frecuencia y puede persistir durante más de 19 días. Estos síntomas se acompañan con mucha frecuencia de deterioro de la función renal. En algunos casos, se ha desarrollado una necrosis tubular aguda que ha llevado a la insuficiencia renal y a la muerte. Todo ello, como ya se ha mencionado previamente, va acompañado de priapismo en el varón y de congestión pélvica en la mujer. A nivel dermatológico, el contacto de la piel con esta sustancia produce su irritación y la formación de ampollas. Además, si la exposición es importante pueden causar toxicidad sistémica con diaforesis — sudoración profusa—, taquicardia, hematuria y oliguria. Respecto a otras posibles complicaciones que conlleva, ya en casos más reducidos, destaca la taquicardia sinusal aunque también han sido descritos efectos mucho más graves como han sido la asistolia o la fibrilación ventricular. El pulmón es un órgano que se afecta con menos frecuencia, pero también se han producido casos de edema pulmonar. A nivel hematológico destaca la policitemia y el síndrome de coagulación intravascular diseminada y a nivel neurológico se han descrito convulsiones, ataxia e hiperreflexia (Karras et al., 1999).

Así pues, habiendo repasado en líneas generales los efectos de este tóxico al que difícilmente podríamos calificar de "afrodisíaco», se puede constatar que la cantaridina no pudo influir de ninguna manera en la muerte del Rey Católico. La literatura científica sobre este tema recoge que los efectos tóxicos de esta sustancia se manifiestan entre las 2 y 10 horas tras la ingesta. En el caso de Fernando II, Pedro Mártir de Anglería dejó escrito que cuando tuvo ese cuadro de vómitos había sido porque le habían administrado la mezcla afrodisíaca y que «nunca más volvió a sentirse en salud». No obstante, pasaron unos ocho meses, entre marzo y noviembre hasta que el humanista apuntó el siguiente síntoma relevante en la evolu- 
ción: la disnea, y más de un año y medio hasta que aparecieron los edemas. Es cierto que la intoxicación por cantaridina podría dar lugar a dificultad respiratoria y a la aparición de edemas — probablemente debidos al deterioro de la función renal y a la pérdida de proteínas por la orina- pero los efectos de la misma se habrían hecho patentes de una forma mucho más precoz en el tiempo. Una última opción sería que hubiera existido el caso de una intoxicación crónica por el elixir de la mosca española. Pese a que no se ha encontrado bibliografía científica al respecto, parece improbable por el largo tiempo, prácticamente tres años, a los que tendría que haberse visto sometido a muchos de los efectos anteriormente descritos. De esta forma, es necesario descartar la acción de un afrodisíaco y apuntar a otras hipótesis con los síntomas guía de los que se disponen.

\subsection{Nuevas hipótesis}

La dificultad respiratoria o disnea fue recogida por Pedro Mártir de Anglería bajo el término «asma» y lo que describió a lo largo de la evolución fue un empeoramiento cuando se encontraba en sitios cerrados. Destaca, por ejemplo, el ataque de disnea brusca en julio de 1515, unos 6 meses antes de la muerte, que fue interrumpido por el guardia nocturno. En el caso de los edemas, descritos por el lombardo como «hidropesía», se afirma que eran progresivos, permanentes y no mejoraban ni con el movimiento ni con el reposo. Estos datos, desde el punto de vista semiológico, podrían orientar a tres orígenes distintos de la patología: corazón, riñón e hígado.

Considerando como posible origen la patología cardíaca, la disnea se manifiesta primero cuando se realiza ejercicio y va evolucionando hasta hacerse de reposo (Laso, 2010). Otras manifestaciones frecuentes son la ortopnea - una intensificación de la disnea al adoptar la posición del decúbito, que mejora al incorporarse- y la disnea paroxística nocturna una crisis de dificultad respiratoria durante el descanso nocturno-. En el caso del Católico se podría interpretar como una crisis de disnea paroxística nocturna lo acontecido en julio de 1515. Esta disnea aparece con frecuencia cuando se produce una congestión pulmonar pasiva, uno de los síntomas cardinales de la insuficiencia cardíaca izquierda, es decir, un deterioro de la función del corazón. En cuanto a la necesidad de Fernando de «estar a cielo abierto», se podría explicar por esa dificultad respiratoria. En una época en la que la ventilación de interiores era deficiente debido al uso de combustibles continuado para calentar e iluminar, los problemas para el 
intercambio de gases se verían agravados. ${ }^{16}$ Por esto mismo, se explicaría la preferencia casi obsesiva por los lugares al aire libre.

Los edemas son la acumulación de líquido en el espacio intersticial, extravasado desde el espacio intravascular. En el caso de la insuficiencia cardíaca, suelen ser predominantes cuando el paciente se encuentra levantado en las extremidades inferiores y después, de no haber mejoría, se generalizan de forma ascendente. En las primeras etapas de la enfermedad, los edemas mejoran con el reposo en decúbito, de tal forma que por la mañana el paciente se despierta con las piernas menos hinchadas y conforme va pasando el día va aumentando el edema. Cuando la insuficiencia cardíaca está más establecida, estos edemas ya no se reabsorben. De ahí, por lo tanto, la aseveración de Pedro Mártir de que el rey no mejoraba ni en reposo ni en movimiento. Los edemas son un signo característico de la insuficiencia cardíaca derecha, con lo cual, se podría establecer que primero comenzó el deterioro de la función de las cámaras izquierdas del corazón (disnea) y al aumentar la presión de la circulación pulmonar se produjo una sobrecarga del ventrículo derecho con la consiguiente insuficiencia cardíaca derecha convirtiéndose así en una insuficiencia cardíaca global (Fauci et al., 2009: 231-236).

Las causas de insuficiencia cardíaca son muy variadas, entre ellas destacan la cardiopatía isquémica, valvulopatías, cardiomiopatías, anemias, hipertiroidismo, sepsis, etc. La alimentación de Fernando el Católico, a falta de estudios al respecto, ha de considerarse la típica de las clases privilegiadas, es decir, muy rica en carne. Esto incrementaría sus posibilidades de sufrir problemas de carácter cardiovascular, como hipertensión o hipercolesterolemia. ${ }^{17}$ Teniendo en cuenta las fuentes manejadas, los principales síntomas fueron la disnea, seguida de los edemas, lo cual encajaría con insuficiencia cardíaca como principal patología responsable de la muerte del Rey Católico.

Respecto a un posible origen renal, destacarían más los edemas que la disnea que en estos casos, suele ser menos intensa que en la insuficiencia

16 De hecho, así sucedía con la momia del infante Sancho de Castilla. Franco, Esteban et al. (2012, p. 38): «El tejido pulmonar fue el más exhaustivamente analizado dado el aspecto patológico que presentaba macroscópicamente. Lo más destacable en las secciones histológicas era la abundante presencia de pigmento antracótico, lo que indica una exposición crónica a humo, probablemente de hogar o chimenea».

17 Sobre este tema, sin ánimo de ser exhaustivos, basten las obras de Flandrin y Montanari (2004); Montanari (1993); García Guatas, Piedrafita, y Barbacil (2013). 
cardíaca y no suelen asociar ortopnea. Cuando el riñón es el órgano que se afecta, puede acompañarse de signos y síntomas de uremia que incluyen inapetencia, disgeusia — regusto metálico o a pescado-, alteración de las características del sueño, dificultad de concentración y acatisia — trastorno del movimiento caracterizado por un sentimiento de inquietud y una necesidad imperiosa de estar en constante movimiento. Los edemas debidos a causa renal, principalmente debidos a la hipoproteinemia — descenso de las proteínas en sangre que se suelen estar perdiendo por la orina-, a veces son más intensos que cuando son generados por motivos cardíacos y pueden afectar a zonas laxas como los párpados o la cara y tienden a ser más intensos por la mañana. Parece, por las descripciones de Pedro Mártir de Anglería, que los edemas se fueron extendiendo y que fue en los últimos momentos cuando se generalizaron. Esta descripción de los edemas probablemente se corresponda mejor con la causa cardíaca además de la disnea, que fue la primera manifestación clínica relevante.

Por último, en una hipotética causa hepática, se considera que la disnea es poco frecuente, salvo si concurre con ascitis - aumento del perímetro abdominal debido a la acumulación de líquido seroso libre en la cavidad abdominal- y muy a menudo coincide con el antecedente de consumo de alcohol. Por lo que se recoge en los escritos de Pedro Mártir de Anglería no parece que este sea el origen más probable.

\section{Conclusiones}

El estudio de monarcas hispanos por causas naturales ha tenido un escaso recorrido historiográfico, quizás más por falta de fuentes solventes que de interés. En el caso de Fernando el Católico, del que se desconocen todavía muchos aspectos de su vida, la historiografía reciente ha seguido dando por válida la explicación que dieron sus contemporáneos respecto a su óbito, siendo esta el suministro de afrodisíacos por parte de su mujer, la reina Germana.

Dentro de los estimulantes de la potencia sexual más comunes, una vez descartados los testículos de toro, la cantaridina - procedente de la mosca española - ha aparecido de manera recurrente como el posible producto. Si bien son obras de escasa fundamentación empírica, a lo largo del presente trabajo se ha desmontado esta posibilidad. Así lo demuestra la larga evolución de la enfermedad de Fernando II; prácticamente fueron tres años lo que sobrevivió a esa supuesta —e improbable — primera ingesta. 
Las fuentes consultadas tienen una información bastante limitada pero importante, ya que los autores coetáneos o posteriores repitieron la información proporcionada por Pedro Mártir de Anglería, fiel espectador de la corte fernandina. Es cierto que existen grandes diferencias en cuanto a paradigmas mentales y representativos entre su época y la actual, pero el cortesano se mostró siempre certero en sus descripciones y apreciaciones médicas. Aunque en ocasiones se ha podido ratificar y completar por otros medios, sigue siendo la principal fuente de conocimiento sobre la evolución de la enfermedad del monarca y no existe otro más completo conocido hasta el momento. Por lo tanto, el diagnóstico se ha efectuado prácticamente siguiendo las percepciones que dejó por escrito el lombardo.

Recopilando los indicios que el citado personaje comunicó a sus distintas amistades epistolares, la disnea y los edemas aparecen como principales síntomas guía de la enfermedad que consumió a Fernando II. Estos pudieron tener, como se ha planteado, tres posibles orígenes: cardíaco, renal y hepático. Teniendo en cuenta el orden de aparición de los dichos síntomas, el fallo cardíaco parece ser la opción más plausible como causante del deterioro físico absoluto en el que pasó sus últimos años de vida el Rey Católico.

\section{REFERENCIAS Bibliográficas}

Alonso Cortés, Narciso, 1951, «Dos médicos de los Reyes Católicos», Hispania: Revista española de historia, 45 (1951): 607-657.

Álvarez, Gonzalo; Francisco C. Ceballos y Celsa Quinteiro, 2009, «The Role of Inbreeding in the Extinction of a European Royal Dynasty», PLoS ONE, 4/4 (2009). Edición digital.

Anglería, Pedro Mártir de, [1956], Epistolario. Madrid. Traducción de José López de Toro. (Colección Documentos inéditos para la historia de España, XI).

Arrizabalaga, Jon, 1994, «La identificación de las causas de muerte en la Europa pre-industrial: algunas consideraciones historiográficas». En Josep BernabeuMestre, Elena Robles González (coords.), Expresiones diagnósticas y causas de muerte. Alicante, Universidad de Alicante: 23-47.

ARrizabalagA, Jon, 2002, «Problematizing retrospective diagnosis in the History of Disease», Asclepio, 54 (2002): 51-70.

Arrizabalaga, Jon, 2006, «El léxico médico del pasado: los nombres de las enfermedades», Panace@: Revista de Medicina, Lenguaje y Traducción, 24 (2006): 242-249.

CABrera SÁnchez, Margarita, 2011, «La muerte de los miembros de la realeza hispánica medieval a través de los testimonios historiográficos», En la España medieval, 34 (2011): 97-132. 
Dormer, Diego José, 1697, Anales de Aragón desde 1525 hasta 1540. Zaragoza.

Fauci, Anthony S., Eugene Braunwald et alii, 2009, Principios de Medicina Interna. I. Madrid, Mc GrawHill.

FlANDRIN, Jean-Louis y Massimo MONTANARI, 2004, Historia de la alimentación. Gijón, Trea.

FranCO, Agustín, Jordi EstebAn et alii, 2012, «Paleoendoscopia: el estudio paleopatológico de la momia de Sancho, hijo del rey Pedro I de Castilla El Cruel (s. XIV)», Medicina clínica, 138 (2012): 37-40.

Galíndez de Carvajal, Lorenzo, 1851, Memorial o registro breve de los Reyes Católicos. Madrid. Edición de Miguel Salvá y Pedro Sainz de Baranda. (Colección Documentos Inéditos para la Historia de España, XVIII.)

García Guatas, Manuel, Elena Piedrafita y Juan Barbacil, 2013, La alimentación en la Corona de Aragón, siglos XIV-XV. Zaragoza, Institución Fernando el Católico.

Gargantilla, Pedro, 2005, Enfermedades de los reyes de España. Los Austrias. De la locura de Juana a la impotencia de Carlos II el Hechizado. Madrid, La Esfera de los Libros.

Gómez GonzÁlez, Jaime, 2004, «La última enfermedad de Isabel la Católica 1451-1504,», Revista Instituto Médico «Sucre», 124 (2004): 82-89.

Guerrero Peral, Ángel Luis, 2009, «Manifestaciones neurológicas de la lepra del rey Balduino IV de Jerusalén», Revista de neurología, 849 (2009): 430-433.

KAmEN, Henry, 2014, «Fernando el Católico, el absolutismo y la Inquisición». En Aurora Egido, y José Enrique Laplana, La imagen de Fernando el Católico en la Historia, la Literatura y el Arte. Zaragoza, Institución Fernando el Católico: 15-27.

Karras, David J., Susan E. Farrell et alii, 1996, «Poisoning from 'Spanishfly' Cantharidin", American Journal of Emergency Medicine (Am J EmergMed), 14 (1996): 478-483.

Laín Entralgo, Pedro, 1998, La historia clínica. Madrid, Triacastela.

LASO, F. Javier, 2010, Introducción a la medicina clínica. Fisiopatología y semiología. Madrid, Elsevier Masson.

Marañón, Gregorio, 1997, Ensayo biológico sobre Enrique IV de Castilla y su tiempo, Madrid, Colección Austral.

Martín Rodríguez, José Luis, 1991, «El rey ha muerto. ¡Viva el Rey!», Hispania: revista española de historia, 151 (1991): 5-39.

Mitchell, Piers D., 2011, «Retrospective diagnosis and the use of historical texts for investigating disease in the past», International Journal of Paleopathology, 1 (2011): 81-88.

Moed, Lisa, Tor A. Shwayder y Mary W. Chang, 2001, «Cantharidin Revisited. A blistering defense of anancient medicine», Archives of Dermatology (ArchDermatol), 137 (2001): 1357-1360. 
MOnTANARI, Massimo, 1993, El hambre y la abundancia: historia y cultura de la alimentación en Europa. Barcelona, Crítica.

Otero Tejero, Ignacio, 2013, Patobiografía. Fernando el Católico. Madrid, Pierre Fabre Ibérica S.A.

PeÑasco, Sandra María, 2013, «La enfermedad vista a través de las relaciones de sucesos», Artifara, 13 (2013): 113-126.

Pérez Monzón, Olga, 2007, «'Quando rey perdemos nunq(u)a bien nos fallamos'. La muerte del rey en la Castilla del siglo XIII», Archivo español de arte, 320 (2007): 379-394.

Presto, Andrew H. III y Edward C. Muecke, 1970, «A dose of Spanish fly», JAMA, 214 (1970): 591-592.

SACHís BANÚs, José, 1927, «La enfermedad y muerte del príncipe don Carlos, hijo de Felipe II», Archivos de medicina, cirngía y especialidades, 306 (1927): 493511.

SAndoval, Prudencio de, 1846, Historia de la vida y hechos del Emperador Carlos V: Máximo, fortísimo, Rey Católico de España y de las Indias, Islas y Tierra firme del mar Océano. I. Madrid, Estudio Tipográfico-Literario de P. Madoz y L. Sagasti.

SAnUto, Marino, 1887, I diari di Marino Sanuto. XXI. Ed. de Rinaldo Fulin et al. Venetia, Marco Visentini.

Torre, Antonio de la, 1944, «Un médico de los Reyes Católicos», Hispania: Revista española de historia, 14 (1944): 66-72.

VENDRELl De Millás, Francisca, 1958, «Relación médica de la enfermedad del rey Fernando de Antequera», Archivo Iberoamericano de Historia de la Medicina y Antropología Médica, 10 (1958): 111-119.

Zurita, Jerónimo, 2005, Historia del rey don Fernando el Católico. De las empresas, y ligas en Italia. Edición electrónica coordinada por J. Javier Iso. Zaragoza, Institución Fernando el Católico. 